\title{
A BIOECONOMIC MODEL OF NONSELECTIVE HARVESTING OF TWO COMPETING FISH SPECIES
}

\author{
D. PUROHIT ${ }^{1}$ and K. S. CHAUDHURI ${ }^{1}$
}

(Received 18 June, 2002)

\begin{abstract}
This paper deals with the combined bioeconomic harvesting of two competing fish species, each of which obeys the Gompertz law of growth. The catch-rate functions are chosen so as to reflect saturation effects with respect to stock abundance as well as harvesting effort. The stability of the dynamical system is discussed and the existence of a bionomic equilibrium is examined. The optimal harvest policy is studied with the help of Pontryagin's maximum principle. The results are illustrated with the help of a numerical example.
\end{abstract}

\section{Introduction}

Harvesting of multispecies fisheries is an important area of study in fishery modelling. The basic ideas related to this field of study were first provided by Clark [7]. Clark also considered the harvesting of one species in a fishery consisting of two competing species. Chaudhuri $[3,4]$ has studied the combined harvesting of two competing species from the standpoint of bioeconomic harvesting and has discussed dynamic optimisation of the harvest policy. Chaudhuri and SahaRay [6] have studied combined harvesting of a prey-predator community with some prey hiding in refuges. The problem of nonselective (that is, combined) harvesting of a prey-predator fishery with infected prey has been studied by Chattopadhyay, Ghosal and Chaudhuri [2]. Pradhan and Chaudhuri [13] developed a dynamic reaction model for harvesting a two-species fishery with taxation as a control instrument. Multispecies harvesting models have also been studied by Silvert and Smith [15], Ragozin and Brown [14], Wilen and Brown [16] and Mesterton-Gibbons $([9,10])$ amongst others.

In almost all of the multispecies fishery models referred to above, the rate of growth was considered to follow the logistic law of growth [7]. In the present paper, we have

\footnotetext{
${ }^{1}$ Department of Mathematics, Jadavpur University, Calcutta-700 032, India; e-mail: jumath@cal.vsnl.net.in.

(C) Australian Mathematical Society 2004, Serial-fee code 1446-181 1/04
} 
considered the Gompertz law of growth because of its superiority over the logistic law of growth as discussed in detail by Pradhan and Chaudhuri [12]. The existence of the possible steady states along with their local stability is discussed. The optimal harvest policy is discussed using the maximum principle due to Pontryagin [11]. A numerical example is taken up to illustrate how the system works.

\section{Formulation of the problem}

The population dynamics of the fishery are modelled by the equations

$$
\begin{aligned}
& \frac{d x}{d t}=F(x, y)-h_{1}(t), \\
& \frac{d y}{d t}=G(x, y)-h_{2}(t),
\end{aligned}
$$

where $x(t)$ and $y(t)$ are the population densities of the two species at time $t$. It is assumed that the growth of each species is governed by the Gompertz law of growth. The harvest rates are $h_{1}(t)$ and $h_{2}(t)$ respectively.

We take

$$
F(x, y)=r x \log (K / x)-\alpha x y
$$

and

$$
G(x, y)=s y \log (L / y)-\beta x y,
$$

where $r, s$ are the intrinsic growth rates (or biotic potentials), and $K, L$ are the carrying capacities of the two species. The interaction terms $-\alpha x y$ and $-\beta x y$ imply that the two species compete with each other for the use of a common resource.

Here the rates of harvest are taken in the form [5]

$$
\begin{aligned}
& h_{1}(t)=\frac{q_{1} E(t) x(t)}{a_{1} E(t)+b_{1} x(t)}, \\
& h_{2}(t)=\frac{q_{2} E(t) y(t)}{a_{2} E(t)+b_{2} y(t)},
\end{aligned}
$$

where $q_{1}$ and $q_{2}$ are the catchability coefficients of the two species, $E$ denotes the effort devoted to their nonselective harvesting and $a_{1}, a_{2}, b_{1}, b_{2}$ are positive parameters of the system.

The catch-rate function generally used in fishery models is of the form $h=q E x$. This is based on the constant CPUE (catch-per-unit-effort) hypothesis [7]. It has several limitations including:

(i) random search for fish; 
(ii) each fish having an equal likelihood of being captured;

(iii) unbounded linear increase in $h$ with $E$ for a fixed $x$; and

(iv) unbounded linear increase in $h$ with $x$ for a fixed $E$.

These unrealistic features are largely removed in (2.1), where $h_{1} \rightarrow\left(q_{1} / a_{1}\right) x$ as $E \rightarrow \infty$ for a fixed value of $x$ and $h_{1} \rightarrow\left(q_{1} / b_{1}\right) E$ as $E \rightarrow \infty$ for a fixed value of $E$. Similarly $h_{2} \rightarrow\left(q_{2} / a_{2}\right) y$ as $E \rightarrow \infty$ for a fixed value of $y$ and $h_{2} \rightarrow\left(q_{2} / b_{2}\right) E$ as $E \rightarrow \infty$ for a fixed value of $E$. Thus the catch-rate function in (2.1) embodies saturation efforts with respect to the effort level as well as stock abundance. Any realistic catch-rate function should exhibit these features. These features also reveal the characteristics of the parameters $a_{1}, a_{2}, b_{1}$ and $b_{2}$. We note that $a_{1}$ and $a_{2}$ are each proportional to the ratio of the stock-level to the catch-rate at higher levels of effort and $b_{1}$ and $b_{2}$ are each proportional to the ratio of the effort level to the catch-rate at higher stock-levels.

Therefore we have the following system of equations:

$$
\begin{aligned}
& \frac{d x}{d t}=r x \log \frac{K}{x}-\alpha x y-\frac{q_{1} E x}{a_{1} E+b_{1} x}, \\
& \frac{d y}{d t}=s y \log \frac{L}{y}-\beta x y-\frac{q_{2} E y}{a_{2} E+b_{2} y} .
\end{aligned}
$$

\section{The steady states}

The possible trivial steady-state points of $(2.2)$ are $P_{1}(0,0), P_{1}(\bar{x}, 0)$ and $P_{2}\left(0, \bar{y}^{*}\right)$. These steady states cannot exist due to the presence of logarithmic functions in (2.2). The nontrivial steady state $P_{3}(\overline{\bar{x}}, \overline{\bar{y}})$, given by $\dot{x}=\dot{y}=0$, is obtained by simultaneously solving the equations

$$
r \log \frac{K}{x}-\alpha y-\frac{q_{1} E}{a_{1} E+b_{1} x}=0
$$

and

$$
s \log \frac{L}{y}-\beta x-\frac{q_{2} E}{a_{2} E+b_{2} y}=0 .
$$

We may solve (3.1) and(3.2) simultaneously for given parameter values to obtain the steady state $(\overline{\bar{x}}, \overline{\bar{y}})$ corresponding to a special effort level.

Eliminating $E$ from (3.1) and(3.2), we have the equation

$$
\frac{b_{1} x(r \log (K / x)-\alpha y)}{q_{1}-a_{1}(r \log (K / x)-\alpha y)}-\frac{b_{2} y(s \log (L / y)-\beta x)}{q_{2}-a_{2}(s \log (L / y)-\beta x)}=0 .
$$

Any point $(x, y)$ on this curve is a nontrivial steady state. We refer to (3.3) as the biological equilibrium path. 


\section{Local stability}

The variational matrix of the system $(4)$ at $P_{3}(\overline{\bar{x}}, \overline{\bar{y}})$ is

$$
V(\overline{\bar{x}}, \overline{\bar{y}})=\left|\begin{array}{cc}
\frac{b_{1} q_{1} E \overline{\bar{x}}}{\left(a_{1} E+b_{1} \overline{\bar{x}}\right)^{2}}-r & -\alpha \overline{\bar{x}} \\
-\beta \overline{\bar{y}} & \frac{b_{2} q_{2} E \overline{\bar{y}}}{\left(a_{2} E+b_{2} \bar{y}\right)^{2}}-s
\end{array}\right| .
$$

The eigenvalues $\lambda_{i}(i=1,2)$ of the system $(2.2)$ at $P_{3}(\overline{\bar{x}}, \overline{\bar{y}})$ are the roots of the following quadratic equation in $\lambda$ :

or

$$
\left|\begin{array}{cc}
\frac{b_{1} q_{1} E \overline{\bar{x}}}{\left(a_{1} E+b_{1} \overline{\bar{x}}\right)^{2}}-r-\lambda & -\alpha \overline{\bar{x}} \\
-\beta \overline{\bar{y}} & \frac{b_{2} q_{2} E \overline{\bar{y}}}{\left(a_{2} E+b_{2} \overline{\bar{y}}\right)^{2}}-s-\lambda
\end{array}\right|=0
$$

$$
\lambda^{2}-(M+N) \lambda+M N-\alpha \beta \overline{\bar{x}} \overline{\bar{y}}=0,
$$

where

$$
M=\frac{b_{1} q_{1} E \overline{\bar{x}}}{\left(a_{1} E+b_{1} \overline{\bar{x}}\right)^{2}}-r \quad \text { and } \quad N=\frac{b_{2} q_{2} E \overline{\bar{y}}}{\left(a_{2} E+b_{2} \overline{\bar{y}}\right)^{2}}-s .
$$

In (4.i), the sum of the roots $=M+N$ and the product of the roots $=M N-\alpha \beta \overline{\bar{x}} \overline{\bar{y}}$. Now

$$
(M+N)>0 \text { if } \frac{b_{1} q_{1} E \overline{\bar{x}}}{\left(a_{1} E+b_{1} \overline{\bar{x}}\right)^{2}}+\frac{b_{2} q_{2} E \overline{\bar{y}}}{\left(a_{2} E+b_{2} \overline{\bar{y}}\right)^{2}}>r+s
$$

and

$$
(M+N)<0 \quad \text { if } \quad \frac{b_{1} q_{1} E \overline{\bar{x}}}{\left(a_{1} E+b_{1} \overline{\bar{x}}\right)^{2}}+\frac{b_{2} q_{2} E \overline{\bar{y}}}{\left(a_{2} E+b_{2} \overline{\bar{y}}\right)^{2}}<r+s
$$

Also

$$
M N-\alpha \beta \overline{\bar{x}} \overline{\bar{y}}>0 \text { if } M N>\alpha \beta \overline{\bar{x}} \overline{\bar{y}}
$$

and

$$
M N-\alpha \beta \overline{\bar{x}} \overline{\bar{y}}<0 \text { if } M N<\alpha \beta \overline{\bar{x}} \overline{\bar{y}}
$$

Considering the various possibilities, we have the following cases.

Case I: The conditions (A) and (C) hold simultaneously. Here we have either (i) $\lambda_{i}>0, i=1,2$ or (ii) the $\lambda_{i}$ 's are complex, $\operatorname{Re} \lambda_{i}>0, i=1,2$. The nontrivial steady state $(\overline{\bar{x}}, \overline{\bar{y}})$ is either an unstable node in (i) or an unstable focus in (ii). Case II: The conditions (A) and (D) hold simultaneously. Here either $\lambda_{1}<0<\lambda_{2}$ or $\lambda_{2}<0<\lambda_{1}$. The steady state $(\overline{\bar{x}}, \overline{\bar{y}})$ is a saddle point. 
Case III: The conditions (B) and (C) hold simultaneously. Here either (i) $\lambda_{i}<0$, $i=1,2$ or (ii) the $\lambda_{i}$ 's are complex, $\operatorname{Re} \lambda_{i}<0, i=1,2$. The steady state $(\overline{\bar{x}}, \overline{\bar{y}})$ is either an asymptotically stable node in (i) or an asymptotically stable focus in (ii).

Case IV: The conditions (B) and (D) hold simultaneously. The conclusion in Case II holds.

Besides the above four cases, the following two cases may arise when $M+N=0$. Case V: The condition (C) together with the condition $M+N=0$ holds. Here the $\lambda_{i}$ 's are complex, $\operatorname{Re} \lambda_{i}=0, i=1,2$. The steady state $(\overline{\bar{x}}, \overline{\bar{y}})$ is a centre.

Case VI: The condition (D) together with the condition $M+N=0$ holds. Here $\lambda_{1}$ and $\lambda_{2}$ are both real, equal in magnitude and of opposite signs. The steady state $(\overline{\bar{x}}, \tilde{\bar{y}})$ is a saddle point.

\section{Bionomic equilibrium}

The bionomic equilibrium is an amalgamation of the concepts of biological equilibrium as well as economic equilibrium. A biological equilibrium is given by $\dot{x}=\dot{y}=0$. The economic equilibrium is said to be achieved when TR (the total revenue obtained by selling the harvested biomass) equals TC (the total cost for the effort devoted to harvesting).

The net economic revenue obtained from the fishery is

$$
\Pi=\mathrm{TR}-\mathrm{TC}=\frac{p_{1} q_{1} x E}{a_{1} E+b_{1} x}+\frac{p_{2} q_{2} y E}{a_{2} E+b_{2} y}-c E,
$$

where $p_{1}$ and $p_{2}$ are the constant prices per unit biomass of the $x$ and $y$ species respectively and $c$ is the constant cost per unit effort. Hence the economic equilibrium is given by

$$
\frac{p_{1} q_{1} x}{a_{1} E+b_{1} x}+\frac{p_{2} q_{2} y}{a_{2} E+b_{2} y}-c=0 .
$$

We refer to (5.1) as the economic equilibrium path. The bionomic solution $\left(x_{b}, y_{b}\right)$ is obtained by solving (3.3) and (5.1) simultaneously for given $E$ and other parameter values.

\section{Optimal harvest policy}

The present value $J$ of a continuous time-stream of revenues is given by

$$
J=\int_{0}^{\infty} e^{-\delta t}\left(\frac{p_{1} q_{1} x}{a_{1} E+b_{1} x}+\frac{p_{2} q_{2} y}{a_{2} E+b_{2} y}-c\right) E(t) d t .
$$


We now intend to maximise (6.1) subject to the state equation (2.2) using Pontryagin's maximum principle [11]. The control variable $E(t)$ is subjected to the constraints $0 \leq E(t) \leq E_{\max }$, where $E_{\max }$ is a feasible upper limit of the effort and it may be a constant or a function of $x$ and $t$. Here $V_{t}=\left[0, E_{\max }\right]$ is the control set.

The Hamiltonian function for the optimisation problem is

$$
\begin{aligned}
H= & e^{-\delta t}\left[\frac{p_{1} q_{1} x}{a_{1} E+b_{1} x}+\frac{p_{2} q_{2} y}{a_{2} E+b_{2} y}-c\right] E(t) \\
& +\lambda_{1}(t)\left[r x \log \frac{K}{x}-\alpha x y-\frac{q_{1} E x}{a_{1} E+b_{1} x}\right] \\
& +\lambda_{2}(t)\left[s y \log \frac{L}{y}-\beta x y-\frac{q_{2} E y}{a_{2} E+b_{2} y}\right] \\
= & {\left[e^{-\delta t}\left(\frac{p_{1} q_{1} x}{a_{1} E+b_{1} x}+\frac{p_{2} q_{2} y}{a_{2} E+b_{2} y}-c\right)-\frac{\lambda_{1} q_{1} x}{a_{1} E+b_{1} x}-\frac{\lambda_{2} q_{2} y}{a_{2} E+b_{2} y}\right] E } \\
& +\lambda_{1}\left(r x \log \frac{K}{x}-\alpha x y\right)+\lambda_{2}\left(s y \log \frac{L}{y}-\beta x y\right)
\end{aligned}
$$

where $\lambda_{1}(t), \lambda_{2}(t)$ are the adjoint variables. The adjoint equations are

$$
\begin{aligned}
\frac{d \lambda_{1}}{d t}=\frac{-\partial H}{\partial x}= & -\left\{e^{-\delta t} \frac{a_{1} p_{1} q_{1} E}{\left(a_{1} E+b_{1} x\right)^{2}}-\frac{a_{1} \lambda_{1} q_{1} E}{\left(a_{1} E+b_{1} x\right)^{2}}\right\} E \\
& -\lambda_{1}\left(r \log \frac{K}{x}-r-\alpha_{1} y\right)-\lambda_{2} \beta_{1} y
\end{aligned}
$$

and

$$
\begin{aligned}
\frac{d \lambda_{2}}{d t}=\frac{-\partial H}{\partial y}= & -\left\{e^{-\delta t} \frac{a_{2} p_{2} q_{2} E}{\left(a_{2} E+b_{2} y\right)^{2}}-\frac{a_{2} \lambda_{2} q_{2} E}{\left(a_{2} E+b_{2} y\right)^{2}}\right\} E \\
& +\lambda_{1} \alpha x-\lambda_{2}\left(s \log \frac{L}{y}-s-\beta x\right) .
\end{aligned}
$$

We now try to derive an optimal equilibrium solution of the problem. For the equlibrium solution, $x$ and $y$ are treated as constants in the subsequent calculations. From the equilibrium equations (3.1) and (3.2), we have

$$
r \log \frac{K}{x}-\alpha y=\frac{q_{1} E}{a_{1} E+b_{1} x} \quad \text { and } \quad s \log \frac{L}{y}-\beta x=\frac{q_{2} E}{a_{2} E+b_{2} y} .
$$

Substituting (6.5) in (6.3) and (6.4), we have

$$
\begin{aligned}
\frac{d \lambda_{1}}{d t} & =-\left[e^{-\delta t} \frac{a_{1} p_{1} q_{1} E}{\left(a_{1} E+b_{1} x\right)^{2}}-\frac{a_{1} \lambda_{1} q_{1} E}{\left(a_{1} E+b_{1} x\right)^{2}}\right] E-\lambda_{1}\left(\frac{q_{1} E}{a_{1} E+b_{1} x}-r\right)+\lambda_{2} \beta y \\
& =-e^{-\delta t} \frac{a_{1} p_{1} q_{1} E^{2}}{\left(a_{1} E+b_{1} x\right)^{2}}-M \lambda_{1}+\lambda_{2} \beta y
\end{aligned}
$$


and

$$
\frac{d \lambda_{2}}{d t}=-e^{-\delta t} \frac{a_{2} p_{2} q_{2} E^{2}}{\left(a_{2} E+b_{2} y\right)^{2}}-N \lambda_{2}+\lambda_{1} \alpha_{1} x .
$$

Eliminating $\lambda_{2}$ from (6.6) and (6.7), we have

where

$$
\frac{d^{2} \lambda_{1}}{d t^{2}}+(M+N) \frac{d \lambda_{1}}{d t}+(M N-\alpha \beta x y) \lambda_{1}=Q_{1} e^{-\delta t},
$$

$$
Q_{1}=\left[\frac{a_{1} p_{1} q_{1} E^{2}(\delta+s)}{\left(a_{1} E+b_{1} x\right)^{2}}-\frac{a_{2} p_{2} q_{2} \beta y E^{2}}{\left(a_{2} E+b_{2} y\right)^{2}}-\frac{a_{1} b_{2} p_{1} q_{1} q_{2} y E^{3}}{\left(a_{1} E+b_{1} x\right)^{2}\left(a_{2} E+b_{2} y\right)^{2}}\right] .
$$

The particular integral of $(6.8)$ is $\left(Q_{1} / R\right) e^{-\delta t}$, where

$$
R=\delta^{2}-(M+N) \delta+(M N-\alpha \beta x y) .
$$

Taking $\lambda_{1}=A e^{\sigma t}, A \neq 0$, to be a trial solution of the homogeneous equation

$$
\frac{d^{2} \lambda_{1}}{d t^{2}}+(M+N) \frac{d \lambda_{1}}{d t}+(M N-\alpha \beta x y) \lambda_{1}=0,
$$

the auxiliary equation is

$$
\sigma^{2}+(M+N) \sigma+(M N-\alpha \beta x y)=0,
$$

having two roots $\sigma_{i}, i=1,2$. Here $\sigma_{1}+\sigma_{2}=-(M+N)$ and $\sigma_{1} \sigma_{2}=M N-\alpha \beta x y$. We now have the following cases.

Case I: The conditions (A) and (C) hold simultaneously. In this case, $\sigma_{1}$ and $\sigma_{2}$ are both either real and negative or complex conjugates with negative real parts. The general solution of $(6.8)$ is $\lambda_{1}(t)=A_{1} e^{\sigma_{1} t}+B_{1} e^{\sigma_{2} t}+\left(Q_{1} / R\right) e^{-\delta t}$. Hence $\lambda_{1}(t) \rightarrow 0$ as $t \rightarrow \infty$. We may similarly prove that $\lambda_{2}(t) \rightarrow 0$ as $t \rightarrow \infty$.

Case II: The conditions (A) and (D) hold simultaneously. Here $\sigma_{1}$ and $\sigma_{2}$ are real and unequal, the negative root having a greater magnitude. Therefore $\lambda_{1}(t) \rightarrow \infty$ as $t \rightarrow \infty$. A similar result holds for $\lambda_{2}$.

Case III: The conditions (B) and (C) hold simultaneously. Here $\sigma_{1}$ and $\sigma_{2}$ are either both real and positive or complex conjugates with positive real parts. Therefore $\lambda_{1}(t) \rightarrow \infty$ as $t \rightarrow \infty$. Similarly, $\lambda_{2}(t) \rightarrow \infty$ as $t \rightarrow \infty$.

Case IV: The conditions (B) and (D) hold simultaneously. Here $\sigma_{1}$ and $\sigma_{2}$ are real and unequal, the positive root having a greater magnitude. Therefore $\lambda_{1}(t) \rightarrow \infty$ as $t \rightarrow \infty$. We note that $\lambda_{2}(t)$ behaves similarly. We therefore find that a finite optimal equilibrium solution exists in Case I only. We then have

$$
\lambda_{1}(t) e^{\delta t}=A_{1} e^{\left(\sigma_{1}+\delta\right) t}+B_{1} e^{\left(\sigma_{2}+\delta\right) t}+Q_{1} / R
$$


Similarly, we have

$$
\lambda_{2}(t) e^{\delta t}=A_{2} e^{\left(\sigma_{1}+\delta\right) t}+B_{2} e^{\left(\sigma_{2}+\delta\right) t}+Q_{2} / R
$$

where

$$
Q_{2}=\left[\frac{a_{2} p_{2} q_{2} E^{2}(\delta+r)}{\left(a_{2} E+b_{2} y\right)^{2}}-\frac{a_{1} p_{1} q_{1} \alpha x E^{2}}{\left(a_{1} E+b_{1} x\right)^{2}}-\frac{a_{2} b_{1} p_{2} q_{1} q_{2} x E^{3}}{\left(a_{1} E+b_{1} x\right)^{2}\left(a_{2} E+b_{2} y\right)^{2}}\right] .
$$

The transversality condition at infinity requires that the shadow prices $e^{\delta t} \lambda_{i}(t)$, $i=1,2$, of the two populations remain bounded as $t \rightarrow \infty([1,7,8])$. To satisfy this condition, we must assume $A_{i}=B_{i}=0, i=1,2$. We then have from (6.9) and (6.10) that $\lambda_{i}(t)=Q_{i} / R=$ constant, $i=1,2$. We also know that the Hamiltonian given in (6.2) must be maximised for $E \in\left[0, E_{\max }\right]$. Assuming that the optimum equilibrium does not occur either at $E=0$ or $E=E_{\max }$ [7], we must therefore have singular control given by

or

$$
\begin{gathered}
\frac{\partial H}{\partial E}=e^{-\delta t}\left(\frac{p_{1} q_{1} x}{a_{1} E+b_{1} x}+\frac{p_{2} q_{2} y}{a_{2} E+b_{2} y}-c\right)-\frac{\lambda_{1} q_{1} x}{a_{1} E+b_{1} x}-\frac{\lambda_{2} q_{2} y}{a_{2} E+b_{2} y}=0 \\
\frac{p_{1} q_{1} x}{a_{1} E+b_{1} x}+\frac{p_{2} q_{2} y}{a_{2} E+b_{2} y}-c=\frac{q_{1} x}{a_{1} E+b_{1} x}\left(\lambda_{1} e^{\delta t}\right)+\frac{q_{2} y}{a_{2} E+b_{2} y}\left(\lambda_{2} e^{\delta t}\right)
\end{gathered}
$$

or

$$
\begin{gathered}
\frac{p_{1} q_{1} x}{a_{1} E+b_{1} x}+\frac{p_{2} q_{2} y}{a_{2} E+b_{2} y}-c=\frac{q_{1} x Q_{1}}{R\left(a_{1} E+b_{1} x\right)}+\frac{q_{2} y Q_{2}}{R\left(a_{2} E+b_{2} y\right)}, \\
\frac{q_{1} x}{a_{1} E+b_{1} x}\left(p_{1}-\frac{Q_{1}}{R}\right)+\frac{q_{2} y}{a_{2} E+b_{2} y}\left(p_{2}-\frac{Q_{2}}{R}\right)=c .
\end{gathered}
$$

For a given effort level, we may obtain the optimal equilibrium solution $\left(x_{\delta}, y_{\delta}\right)$ by solving (3.3) and (6.11) simultaneously.

As $\delta \rightarrow \infty$, we may easily check that both $Q_{1} / R$ and $Q_{2} / R$ tend to zero. Then (6.11) yields the result

$$
\frac{p_{1} q_{1} x_{\infty}}{a_{1} E+b_{1} x_{\infty}}+\frac{p_{2} q_{2} y_{\infty}}{a_{2} E+b_{2} y_{\infty}}-c=0
$$

or $\Pi\left(x_{\infty}, y_{\infty}, E\right)=0$. This implies that the economic rent is fully dissipated when the discount rate is very high. We thus have an open-access fishery condition as $\delta \rightarrow+\infty$.

Our control problem, therefore, possesses an equilibrium solution that satisfies the necessary conditions of the maximum principle. However, this solution has the following two limitations:

(i) there is no obvious economic interpretation of this solution;

(ii) it is too difficult to find the optimal approach path consisting of some combination of bang-bang controls and nonequilibrium singular controls. 
TABle 1.

\begin{tabular}{lcc}
\hline & $x$ & $y$ \\
\hline Steady state & 80.53 & 53.33 \\
Binomial equilibrium & 94.81 & 27.91 \\
Optimal equilibrium & 32.97 & 31.53 \\
\hline
\end{tabular}

These difficulties were faced and pointed out by Clark [7] even in his simple model of the combined harvesting of two ecologically independent fish populations where the catch-rate functions were based on the simple CPUE hypothesis. Compared to Clark's model, the present model is much more complicated and hence we are compelled to restrict our attention to the optimal equilibrium solution only with singular control.

\section{Numerical example}

Let $r=1.5, s=0.9, K=1000, L=800, q_{1}=0.9, q_{2}=0.8, \alpha=0.07$, $\beta=0.03, p_{1}=14, p_{2}=18, a_{1}=20, a_{2}=16, c=8, b_{1}=2, b_{2}=4.0, \delta=4.71$ and $E=10$ in appropriate units.

Using these parameter values, we numerically determine the solutions for the steady state $(\overline{\bar{x}}, \overline{\bar{y}})$, bionomic equilibrium $\left(\bar{x}_{b}, \bar{y}_{b}\right)$ and optimal equilibrium $\left(x_{\delta}, y_{\delta}\right)$ using the Newton-Raphson method. The results are given in Table 1.

The steady-state solution $(80.53,53.33)$ is obtained by solving (3.1) and (3.2) corresponding to a given effort level $E$. We examine each case from (A) to (D) and find that conditions (B) and (D) are satisfied simultaneously. Hence Case IV holds and the steady state $(\overline{\bar{x}}, \overline{\bar{y}})$ is a saddle point.

The bionomic equilibrium $(94.81,27.91)$ is obtained by solving (3.3) and (5.1), and the optimal equilibrium solution $(32.97,31.53)$ is given by $(3.3)$ and $(6.11)$.

\section{References}

[1] K. J. Arrow and M. Kurz, Public investment. The rate of return and optimal fiscal policy (Johns Hopkins Press, Baltimore, 1970).

[2] J. Chattopadhyay, G. Ghosal and K. S. Chaudhuri, "Nonselective harvesting of a prey-predator community with infected prey", Korean J. Comput. Appl. Math. 6 (1999) 601-616.

[3] K. S. Chaudhuri, "A bioeconomic model of harvesting a multispecies fishery", Ecol. Model. 32 (1986) 267-279.

[4] K. S. Chaudhuri, "Dynamic optimization of combined harvesting of a two-species fishery", Ecol. Model. 41 (1987) 17-25.

[5] K. S. Chaudhuri and T. Johnson, "Bioeconomic dynamics of a fishery modelled as a S-system", Math. Biosci. 99 (1990) 231-249. 
[6] K. S. Chaudhuri and S. SahaRay, "On the combined harvesting of a prey-predator system", J. Biol. Syst. 4 (1996) 373-389.

[7] C. W. Clark, Mathematical bioeconomics: the optimal management of renewable resources (Wiley, New York, 1976).

[8] H. Halkin, "Necessary conditions for optimal control problems with infinite horizons", Econometrica 42 (1974) 267-272.

[9] M. Mesterton-Gibbons, "On the optimal policy for combined harvesting of independent species", Nat. Res. Model. 2 (1987) 107-132.

[10] M. Mesterton-Gibbons, "On the optimal policy for the combined harvesting of predator and prey", Nat. Res. Model. 3 (1988) 63-90.

[11] L. S. Pontryagin, V. S. Boltyanskii, R. N. Gamkrelidge and E. F. Mishchenko, The mathematical theory of optimal processes (Wiley, New York, 1962).

[12] T. Pradhan and K. S. Chaudhuri, "Bioeconomic modelling of a single species fishery with Gompertz law of growth", J. Biol. Syst. 6 (1998) 393-409.

[13] T. Pradhan and K. S. Chaudhuri, "A dynamic reaction model of a two-species fishery with taxation as a control instrument: a capital theoretic analysis", Ecol. Model. 121 (1999) 1-16.

[14] D. L. Ragozin and G. Brown, "Harvest policies and non-market valuation in a predator-prey system", J. Environ. Eco. Mgmt 12 (1985) 155-168.

[15] W. Silvert and W. R. Smith, "Optimal exploitation of a multispecies community", Math. Biosci. 33 (1977) 121-134.

[16] J. Wilen and G. Brown, "Optimal recovery paths for perturbations of trophic level bioeconomic systems", J. Environ. Eco. Mgmt 13 (1986) 225-234. 